\title{
Recuperación Ósea en Procedimientos de Reconstrucción y Colocación de Implantes
}

\author{
Osseous Recovery in Implant Insertion and Pre Implant Reconstructions
}

",**:Sergio Olate; "*Greison Rabelo de Oliveira; **Miguel Jaimes; **Jose Ricardo de Albergaria-Barbosa

OLATE, S.; DE OLIVEIRA, G. R.; JAIMES, M. \& BARBOSA J. R. A. Cicatrización ósea en procedimientos de reconstrucción y colocación de implantes. Int. J. Morphol., 25(3):649-657, 2007.

RESUMEN: La implantología ha evolucionado rápidamente en los ultimos 40 años y el empleo de biomateriales en la recuperación de tejidos es cada vez más frecuente. Por esta razón, es necesario conocer el proceso de reparación y la reacción producida entre los los tejidos huéspedes y los biomateriales a nivel histológico, ya que ello contribuirá al éxito, en el largo plazo, de los tratamiento rehabilitadores. Clínicamente, se ha podido observar la efectividad de diferentes compuestos, pero cuando han sido analizados histológicamente, han demostrado algunas variaciones que, muchas veces, las hacen inaceptables para la terapia odontológica. Los objetivos de esta revisión es conocer la forma en que se produce la cicatrización ósea en torno a diferentes biomateriales y como se desarrolla la cicatrización ósea en rehabilitación sobre implantes con diferentes protocolos de carga.

PALABRAS CLAVE: Cicatrización ósea; Biomateriales; Implantes.

\section{INTRODUCCIÓN}

Una de las situaciones que, con altos indicadores, hoy en día afecta a todo el mundo es la elevada cantidad de perdida dentaria (Petersen \& Yamamoto 2005, Olate et al., 2006) de forma que la implantología se desarrolla hoy ante una gran necesidad de la población de recuperar función y estética perdidas, además de las exigencias que plantean los pacientes sobre tratamientos cada vez mas rápidos.

También la reconstrucción ósea de maxilares con el uso de biomateriales se ha transformado hoy en una alternativa terapeutica viable y predecible para determinado tipo de defectos óseos. Sin embargo, estas exigencias de recuperación del volumen óseo mediante procedimientos de injertos también viene a modificar, en buena parte de los casos, la estructura ósea que estará en contacto con el implante de titanio (Scarano et al., 2006).

De igual manera, la rehabilitación con implantes oseointegrados ha tenido importantes avances en materia de estructuras, diseños y superficies, entre otras. En gran parte debido a esto, los tiempos de carga de los implantes y los protocolos quirúrgicos han sufrido modificaciones que per- mitirían mantener e incluso mejorar la calidad de los tratamientos con menores tiempos de trabajo (Morton et al., 2004).

Un tratamiento exitoso debe basarse en la predictibilidad del procedimiento. En base a esa premisa, la cronología y mecanismos celulares involucrados en la fisiopatología de la reparación ósea, además de la forma en que éstos se desarrollan en presencia de implantes o injertos asociados son el fundamento para la estabilidad a largo plazo y, en definitiva, para el éxito clinico de estos tratamientos. La carga de los implantes también ha sufrido modificaciones a lo largo de los años, donde algunas veces no se ha obtenido el fundamento biológico que sustente el éxito o fracaso de éstos (Szmukler-Moncler et al., 2000).

Los objetivos de esta revisión son identificar los biomateriales más empleados en reconstrucción con injertos previo a la colocación de implantes, reconocer la forma en que se incorporan biofuncionalmente e identificar cómo las variaciones de los tipos de carga en implantología interfieren en la recuperación ósea.

\footnotetext{
* Departamento de Odontologia Integral, Facultad de Medicina, Universidad de La Frontera, Chile.

** Departamento de Diagnóstico Oral, Área de Cirurgia Buco Maxilo Facial, Faculdade de Odontologia de Piracicaba, Universidade Estadual de Campinas, Brasil.
} 


\section{Injertos Óseos}

Los injertos óseos como método de recuperación del volumen óseo perdido, se han caracterizado por un constante crecimiento en torno a los biomateriales y a la forma y momento en que se deben emplear (Cortez et al., 2004). No obstante, factores locales como la estructura anatómica del lecho receptor y las características vasculares, entre otras, son de mucha importancia al momento de evaluar la reparación ósea (Canalis et al., 1988). Consecuentemente, diferentes sistemas terapéuticos que potencian esta reparación han sido empleados en pacientes con osteoporosis, y que podrían ser utilizados en tratamiento de la enfermedad periodontal y en regeneración ósea para la recuperación de volumen óseo (Horiuchi \& Maeda, 2006).

De este modo, existe una gran variedad de materiales utilizados en la reconstrucción de maxilares, siendo agrupados en relación a su origen en cuatro tipos distintos (Grageda, 2004):

1) Autoinjertos: hueso transplantado de otra parte del cuerpo (intraoral o extraoral) del mismo paciente al lecho receptor.

2) Aloinjertos: material obtenido de sujetos de la misma especie, y que son procesados por métodos físico y químicos, para permitir su incorporación en el lecho receptor del paciente; dentro de éstos están DFDBA (hueso desmineralizado congelado deshidratado), FDBA (hueso congelado deshidratado), entre otras, BMP (proteína morfogenética).

3) Aloplásticos: son sustitutos óseos sintéticos, cerámicas o polímeros generalmente, con diferentes características unos y otros. Ejemplos de este grupo son la HA (hydroxiapatita), TCP (fosfato tricálcico), sulfato de calcio o su combinación.

4) Xenoinjertos: material obtenido de especies no humanas, procesadas a fin de retener el contenido mineral óseo. En este grupo se encuentra Bio-Oss (hidroxiapatita bovina), bBMP (proteína ósea morfogenética bovina), entre otras.

A pesar de la creciente investigación asociada al desarrollo y uso de biomateriales no autogeneos, éstas aún no han conseguido reproducir las mismas características del propio hueso donante del paciente, hasta ahora considerado "gold standart" en este tipo de procedimientos (Moore $e t$ al., 2001). En este sentido, además de la osteoinducción caracterizada por Urist et al. el año 1983, como el mecanismo que estimula la nueva formación ósea, se persigue también la osteoconducción y osteogeneración, al momento de evaluar un biomaterial. A pesar de ello, la poca cantidad de hueso en el caso de los sitios donantes intraorales, la morbilidad del sitio donante y la necesidad de aumentar los procedimientos quirúrgicos, fundamentan la búsqueda de nuevos compuestos que puedan ser utilizados con fines similares (Grageda).

Biomateriales sintéticos y orgánicos han sido utilizados em diferentes tratamientos quirúrgicos reconstructivos en modelos experimentales animales y también en humanos.

Bio-Oss es un biomaterial a base de hueso bovino desproteinizado con $75-80 \%$ de porosidad y un cristal de $10 \mathrm{~mm}$ de diámetro en forma de gránulos corticales (Degidi et al., 2005). El comportamiento de Bio-Oss ha demostrado efectividad en las evaluaciones clínicas realizadas. Por su naturaleza particulada, debe emplearse junto a algún elemento que funcione como vehículo, mejorando así su adaptación en el lugar de colocación. Frecuentemente este material es empleado en procedimientos de levantamiento de seno maxilar, siendo uno de los rellenos óseos de elección.

A pesar de ello, la respuesta de Bio-Oss en la formación de nuevo hueso es menor a la respuesta entregada por el autoinjerto de hueso particulado visto en evaluaciones morfométricas (Jensen et al., 2005), presentando también una pobre inducción para la formación de osteoblastos (Kübler et al., 2004). Contrariamente, la cantidad de tejido mineralizado con el uso de este biomaterial es mayor a la presentada por otros (Jensen et al.).

Otro tipo de hueso orgánico bovino potenciado con proteína morfogenética del mismo origen, demostró incapacidad en la neoformación ósea, mostrando formación parcial de hueso, presencia de tejido fibroso y zonas radiolúcidas en las zonas injertadas (Serra e Silva et al., 2006). El estudio de Queiroz et al. (2006) demostró buen comportamiento de los injertos de hueso bovino, agregando el hecho de que sería necesaria la presencia de membranas cuando se emplean injertos, para asegurar la ausencia de colapso del tejido blando que circunda el defecto. Por su parte, Froum et al. (2004) encontraron que indistintamente del uso de relleno de hidroxiapatita o hueso bovino anorgánico, el empleo de membranas contribuye a la mantención y respuesta de los injertos, aunque eso no cambiaría las propiedades biológicas de tales materiales.

b-TCP es un material cerámico actualmente en uso. El comportamiento de este material ha sido similar al mostrado por los autoinjertos en cuanto a la formación de nuevo hueso (Jensen et al.) (Tabla I). Estudios histológicos e histomorfométricos no tienen diferencia estadística en la 
reparación ósea de autoinjertos de cresta iliaca comparados con b-TCP, presentando una densidad ósea muy similar (Suba et al., 2006). Consecuentemente, los resultados regenerativos de b-TCP en defectos exclusivamente verticales, son superiores a los presentados por Bio-Oss, sumándose a ello los mejores patrones de reabsorción de bTCP por sobre Bio-Oss (Tamimi et al., 2006) (Tabla II).

Tabla I. Porcentaje de hueso nuevo ocupando defecto óseo creado en cerdos.

\begin{tabular}{lcccc}
\hline & 4 semanas & DE & 8 semanas & DE \\
\hline Autoinjerto & $39,4 \%^{*}$ & 5,1 & $54,4^{* *}$ & 3,9 \\
B-TCF & $36,4 \%$ & 4,3 & $57,4^{* *}$ & 5,3 \\
Bio-Oss & $24,6 \%$ & 3,5 & 41,6 & 4,7 \\
\hline
\end{tabular}

significativamente maior que b-TCF e Bio-Oss (Jensen et al., 2005).

*** significativamente maior que Bio-Oss (Jensen et al., 2005).

Tabla II. Porcentaje de hueso nuevo ocupando defectos realizados en ratones a las 4 semanas solo considerando el crecimiento óseo vertical.

\begin{tabular}{lcccc}
\hline & Aumento & DE & Material no reabsorvido & DE \\
\hline Bio-Oss & $11,7 \%$ & 2,4 & $37,2 \%$ & 5,5 \\
DTG & $16,7 \%^{*}$ & 4,9 & $22,4 \%^{* *}$ & 8,5 \\
\hline
\end{tabular}

* significativamente maior que Bio-Oss (Tamimi et al., 2006).

** significativamente maior que Bio-Oss (Tamimi et al., 2006).

DTG $=$ fosfato monocálcico $(0,8 \mathrm{~g})+\mathrm{b}-\mathrm{TCP}(1,4 \mathrm{~g})+$ solución acuosa ácido glicólico. Polvo - líquido = 1,7 g/l.

El cemento a base de fosfato de calcio, distinto en estructura química a b-TCP, demostró en estudios histomorfométrico y radiográfico, que a 12 semanas post quirúrgicas, presenta poca y defectuosa neoformación ósea, presentando también respuestas inflamatorias importantes. El injerto con material a base de hidróxido de calcio fue estadísticamente superior en el proceso de osteogénesis (Moghadam et al., 2004).

Frente al fracaso de estos biomateriales, Hing et al. (2007) los atribuyen a tres causas: 1) insuficiente persistencia de actividad osteoconductiva, 2) temprana desestabilización de la aposición ósea y 3) estimulación de respuesta inflamatoria que eleva los niveles de degradación de partículas y la actividad fagocitaria. También señalan una pobre respuesta de b-TCF, fosfato de calcio y sulfato de calcio, siendo esta última la que presentó peor desarro$1 \mathrm{lo}$, con un $80 \%$ del injerto perdido a las 3 semanas.

La hidroxiapatita, material orgánico base de la estructura ósea mineral fue sometida a evaluación durante seis meses, presentando $100 \%$ de reabsorción y muchas similitudes en la reparación ósea cuando se comparó con los autoinjertos (Thorwarth et al., 2005)
Estudiando múltiples biomateriales, Scarano et al. encontraron entre un 3\% y un 35\% de permanencia de partículas de biomaterial a 6 meses de evaluación, donde los mejores indicadores fueron obtenidos por autoinjertos. No obstante, los autores concluyeron que es seguro el manejo de estos biomateriales en regeneración ósea previo a la colocación de implantes, mediante una evaluación de carga protésica durante 3 años.

\section{Reparación Ósea en Reconstrucciones de Reborde Alveolar Previo a la Colocación de Implantes.}

Diferentes eventos celulares de variada complejidad son conocidos del proceso de reparación tecidual, pero una buena parte de ellos aún no tienen claridad de la forma en que tienen que ser empleadas en la modificación o la variación temporal de la reparación ósea (Issa et al., 2006a, Issa et al., 2006b, Miura et al., 2006). No obstante, es confirmada por algunos estudios, la importante función de la angiogénesis y vasculogénesis en la reparación de tejidos (Liu et al., 2006). La angiogénesis, definida simplemente como la activación de células endoteliales pre-existentes, y la vasculogénesis, como la formación de nuevos vasos sanguíneos desde células progenitoras endoteliales derivadas de la matriz ósea, tienen importantes implicancias en la reparación tisular y su empleo en regeneración en el futuro es muy esperado por la comunidad científica (Madeddu, 2005, Roethy et al., 2001).

La respuesta de los tejidos al estrés biológico y mecánico es mediada por diferentes factores vasculares y angiogénicos, donde uno de los más importantes es el factor de crecimiento vascular endotelial (VEGF) (Brown \& Hudlicka, 2003). Armir et al. (2006a) observaron en biopsias de pacientes mayores en los que se formó poca cantidad de hueso, que existía también una disminuida formación de vasos sanguíneos, determinando con eso, que una media de distracción osteogénica menor a la utilizada permitía la formación de mayor cantidad de vasos sanguíneos. Otro reporte de Armir et al. (2006b) con los mismos sujetos, demostró una correlación positiva entre densidad y volumen óseos y la presencia de vasos sanguíneos y presencia de tejido conectivo, pero no pudieron determinar correlación entre densidad ósea y tamaño de los vasos sanguíneos. Otro estudio sugirió también similitud en la respuesta vascular entre autoinjertos e injertos aloplásticos, siendo que el primero presentaba una rápida formación vascular. (Piattelli et al., 2002)

Se ha visto que el proceso inflamatorio previo no es necesario para el desarrollo de la angiogénesis, pero si es requerido la presencia de quimiokinas, glicosaminoglicanos (GAGs) y algunos factores de creci- 
miento (Rosenkilde \& Schwartz, 2004; Winet, 1996). Otras investigaciones, también conducen a que las células derivadas del hueso medular mejoran la reparación de tejido por la producción de factores de crecimiento específicos (Liu et al.) y su uso terapéutico se encuentra en una amplia línea de investigación (Roethy et al.). Está definido que la estabilidad de los elementos injertados es crítico en la formación de vasos sanguíneos, tejido fibroso y óseo, donde la movilidad produce un aumento de tejido blando y retarda la cicatrización (Lienau et al., 2005). Otra situación en investigación consiste en el potencial que presentan las "stem cells" derivadas del hueso esponjoso y su capacidad en la regeneración de tejidos y especialmente en la neogénesis vascular, donde un nuevo tipo de biomaterial se podría evaluar (Miura et al.; Li et al., 2003).

Un estudio realizado en 40 ratones con defectos creados en sus calotas, evaluó la reparación tisular utilizando 5 membranas comerciales y 3 membranas experimentales, observando el modelo de angiogénesis a las 2, 8 y 24 semanas. Mediante análisis de inmunohistoquímica, demostró un patrón de relación directa entre biodegradación de la membrana y angiogénesis; a las 24 semanas, cuando la degradación de la membrana está casi completa, los valores de angiogénesis tienden a cero (Schwarz et al., 2006). Ellos observaron también que la angiogénesis tenía directa relación con el tamaño y distribución de las porosidades de la membrana empleada; por ejemplo, a las 24 semanas, los defectos trabajados con Ossix ${ }^{\circledR}$ no presentaron ninguna formación vascular, mientras que todas las membranas testadas sí presentaron formación de vasos sanguíneos.

Kleinheinz et al. (2005) evaluaron la incorporación de rhVEGF165 al colágeno tipo I en defectos creados; los análisis fueron realizados a los 3, 7, 14 y 28 días, mediante exámenes histológico e inmunohistoquímico. Los resultados revelaron un patrón de crecimiento vascular en el frente, seguido de osteogénesis, con un crecimiento vascular permanente por 14 días. Después de ese momento, sólo el grupo de estudio con rhVEGF165, mantuvo su crecimiento y posterior osteogénesis. Esto concuerda con los resultados presentados por Huang et al. (2005) quienes demostraron que la presencia de factores angiogénicos se relacionaba directamente con la cantidad de neogeneración ósea.

Similares resultados presentaron el grupo de Weiss et al. (2005); la VEGF fue de aumentada y mantenida expresión en casos de distracción osteogénica, siendo mayor que en casos de fractura y osteosíntesis. Con esto se puede hipotetizar que el patrón mecánico de tracción tiene importancia en el estimulo de VEGF. Por otra parte, la angiogenina fue de expresión sólo en el momento de la cirugía, descendiendo rápidamente en el grupo de distracción y manteniéndose en el grupo de osteosíntesis. Estos resultados concuerdan con el hecho de que una rápida vascularización de tejido conjuntivo en un defecto óseo resulta en una rápida formación ósea, donde un defecto de mayor tamaño tendría una recuperación ósea más rápida además, teniendo relación también la expresión de la fosfatasa alcalina (Nishimura et al., 2004). La expresión de la fosfatasa alcalina es mayor en injertos de hueso particulado y en maxila, al compararlo con injertos en bloque (Gruber et al., 2005) y su presencia se relaciona con mejores condiciones para la generación de osteoblastos (Kübler et al.). Gruber et al. también demostraron que la proteína morfogenética (BMP-2, BMP-6 y BMP-7) contribuye a una mayor expresión de la fosfatasa alcalina.

\section{Tiempos de Carga de Implantes Oseointegrados.}

Los tiempos de carga han tenido, históricamente, muchas variaciones desde que Bränemark (1983) estipulara los primeros protocolos de carga con aparatos protésicos sobre implantes oseointegrados; actualmente, son diversos autores los que se involucran en la definición de éstos (Aparicio et al. 2003; Wang et al., 2006, Cochran et al., 2004).

En este sentido, fue Albrektsson et al. (1981) quienes establecieron las condiciones para la rehabilitación sobre implantes. Albrektsson e Bränemark señalaban que para el éxito de implantes, se debían respetar los siguientes puntos: 1) mínimo trauma quirúrgico, 2) obtención de estabilidad primaria, 3) ausencia de infección y 4) ausencia de micromovimiento. Este protocolo también exigía que el implante fuera rehabilitado después de algunos meses, tiempo en el que tenía que estar bajo la mucosa y sin carga. Ellos también indicaban las razones para no permitir carga inmediata o temprana en implantes, y que eran: 1) carga prematura puede alcanzar formación de tejido fibroso en lugar de tejido óseo, 2) el hueso necrótico de los límites de la preparación no puede soportar cargas y requiere de nueva formación ósea. (Albrektsson et al.; Bränemark).

En el mismo año que Bränemark escribía sobre la carga tardía de implantes, Schroeder et al. comprobaron que el uso de carga inmediata en sobredentaduras era posible en un seguimiento promedio de 17,5 meses con un 98\% de éxito (Szmukler-Moncler et al.). Idénticos resultados fueron obtenidos por Chiapasco et al. (1997), demostrándolo también en pacientes con reconstrucciones de hueso autógeno. Debido a esto, el protocolo de carga inmediata en sobredentaduras mandibulares se encuentra bien definido y documentado (Cochran et al.; Gapski et al., 2003; Szmukler-Moncler et al.), mientras que para 
otros procedimientos se sugieren "cuidados" no bien descritos (particularmente en la carga inmediata de implantes unitarios), especialmente en el remplazo en zona de incisivos y molares, en relación también a la calidad ósea receptora (Wang et al.).

Existen pocos estudios en el área de carga inmediata y con datos insuficientes la mayoría de ellos (Nkenke \& Fenner, 2006), lo cual implica que las indicaciones para la carga inmediata aún no pueden ser complatemente definidas. Además de eso, existe poca evidencia de los factores de riesgo (como la carga oclusal excesiva, la movilidad o micromovilidad del implante, entre otras) asociados a la carga inmediata de implantes (Hobkirk \& Wiskott, 2006) lo que también dificulta su inserción como protocolos definidos. Gapski et al. sugieren, no obstante, que las características de la cirugía, del huésped, del diseño y tipo de implante, y finalmente la oclusión pueden influenciar en los resultados a largo plazo.

En el caso de edentulismo parcial, no existe protocolo definido para carga inmediata. Sin embargo, esta tendería a asociarse a: (1) ausencia de parafunciones, (2) uso de implante con superficie rugosa y roscada y (3) estabilidad primaria del implante, relacionado íntimamente con el torque de inserción (Ottoni et al., 2005, Schincaglia et al., 2007). Estos conceptos se asocian a la estabilidad primaria, la cual tendría relevancia en la cicatrización de la fijación implantaria (Nkenke et al., 2005a). Clínicamente, los reportes de éxito en implantes de carga inmediata unitarios son alentadores, superando el $90 \%$ en la mayoría de los casos (Degidi \& Piattelli, 2005, Schincaglia et al., Ericsson et al., 2000; Lorenzoni et al., 2003).

De esta forma, la carga inmediata definida como la restauración protetica de un implante realizada antes de 48 horas de colocado en su respectiva posición ósea y con contacto con el diente antagonista (Cochran et al.), ha tenido un importante reconocimiento, toda vez que las investigaciones publicadas se encuentran respaldando su uso en la práctica clínica.

Realizando estudios histológico e inmunohistoquímico en mandíbulas de cerdos, Meyer et al. (2003) demostraron en evaluaciones de hasta 14 días ,que la forma de la estructura ósea y la calidad de la misma no difería entre implantes de carga inmediata e implantes sin carga; y que al pasar este tiempo de evaluación no existen diferencias en el modelo celular de crecimiento o en la mineralización ósea en implantes de carga inmediata o sin carga. Los autores hipotetizan sobre la forma cónica del implante utilizado que aseguraría la posibilidad de contacto óseo en toda su extensión.
Sin embargo, el estudio de Traini et al. (2005) demostró que la carga inmediata tiene diferentes orientaciones de las fibras colágenas al compararlas con implantes sin carga. De esta forma, un implante de carga inmediata tiene más fibras transversales que longitudinales, presentando diferencia estadística significativa. Por otra parte, la cantidad de fibras colágenas fue mucho menos en implantes de carga inmediata que en implantes no cargados. De esta forma, y siendo aún controversial, existiría una relación entre mayor nivel de fibras colágenas transversales y una cortical ósea mayor de volumen (Kalmey \& Lovejoy, 2002). Junto a las características de reparación ósea, el diseño del implante y la ubicación de éste tendría importancia en la distribución de las fuerzas en el hueso (Hanson \& Werke, 2003, Akça et al., 2005) y, por tanto, en las modificaciones de la arquitectura ósea (Nkenke et al., 2003). Coincidentemente, el estudio de McMillan et al. (2000) demostró que las características morfológicas macro y micro de los implantes son importantes en el momento de evaluar la densidad ósea peri - implantar y también la densidad ósea entre las roscas del implante.

A pesar de las variaciones en la distribución de fibras, histológicamente, no se observaron mayores diferencias óseas entre implantes cargados de forma inmediata y los cargados de forma tardía (Nkenke et al., 2005b). El grupo de Nkenke et al. (2003) también demostó que la aposición de tejido mineral es prácticamente igual entre implantes de carga inmediata e implantes no cargados, siendo la cantidad de hueso en contacto con implantes similar en grupos de carga inmediata y sin carga (cerca de $77 \%$ de implante en contacto). Las densidades del hueso peri-implantar y del hueso en contacto con la rosca del implante no mostraron diferencias entre los dos grupos. En este estudio, el grupo de casos fue realizado con 4 implantes unidos con una prótesis fija, concluyendo que la fijación rígida de los implante es un factor crucial para el éxito de los mismos, ya que el fracaso de unos de ellos ocurrió cuando la fijación fue perdida y los implantes presentaron movilidad y contacto con tejido fibroso. Esa fijación con estructuras protésicas permite una movilidad menor a $150 \mu \mathrm{m}$, límite permitido para asegurar el éxito de implantes ósteointegrados, según Szmukler-Moncler et al., pero que en carga inmediata debe ser menor a $30 \mu \mathrm{m}$ según Kawahara et al. (2003). Esa movilidad aumentada se ha relacionado con la presencia de tejido fibroso peri implantar y con la presencia de macrófagos en el caso de implantes perdidos (Olmedo et al., 2003). A pesar de que Cecchianato et al. (2004) mostraron que la reparación ósea aparentemente es independiente de la técnica quirúrgica de implantes sumergidos o no sumergidos, tiene que ser realizado un procedimiento poco traumático para asegurar esa ausencia de movilidad. 


\section{CONCLUSIÓN}

Ya existe claridad de una parte de la secuencia biológica para la cicatrización ósea, que permite el desarrollo de algunas herramientas para la práctica implantológica, así como también la modificación de algunas existentes. La reconstrucción pre implantar de pequeños a moderados defectos debe dirigirse ahora, a potenciar la osteoinducción más que a la recuperación del volumen para asegurar la compatibilidad biologica a largo plazo de las rehabilitaciones. En este sentido, el hueso proveniente de "stem cells" puede contribuir a la formación de nuevos injertos, junto a factores que estimulen la angiogénesis y vasculogénesis, elementos claves en la neoformación ósea. Por otra parte, al no existir diferencias histológicas relevantes en la cicatrización de los implantes con carga convencional y los implantes de carga inmediata, este protocolo quirúrgico debe estudiarse en investigaciones prospectivas a largo plazo y permitir un protocolo quirúrgico - protésico como tratamiento definitivo.

OLATE, S.; DE OLIVEIRA, G. R.; JAIMES, M. \& BARBOSA J. R. A. Osseous recovery implant insertion and pre implant reconstructions. Int. J. Morphol., 25(3):649-657, 2007.

SUMMARY: Implantology had experienced a rapid evolution in the last 30 years and the use of biomaterials on tissue recuperation has been more frequent; for this reason it is necessary to know the repaired process and histological reaction between the hosted tissue and the biomaterials, thereby determining the success of the therapy in the long time. Clinically, it has been to possible to see the effects of different biomaterials, but after an histological analyses, has been demonstrated some variations that make them unaccepted for dental treatment. The aim of this review is to report the way of the bone cicatrisation with different biomaterials and the form in which it is developed this cicatrisation on the rehabilitation with implants with different load protocols.

KEY WORDS: Osseous cicatrization; Biomaterials; Implants.

\section{REFERENCIAS BIBLIOGRÁFICAS}

Albrektsson, T.; Bränemark, P.I.; Hansson, H. A. \& Lindstrom, J. Osseointegrated titanium implants. Requirements for ensuring a long-lasting, direct bone-to-implant anchorage in man. Acta Orthop. Scand., 52 (2):155-70, 1981.

Akça, K.; Akkocaoglu, M.; Cömert, A.; Tekdemir, I. \& Çehreli, M. C. Human ex vivo bone tissue strains around immediately loaded implants supporting maxillary overdentures. Clin. Oral Impl. Res., 16:715-22, 2005.

Aparicio, C.; Rangert, B. \& Sennerby, L. Immediate/early loading of dental implants: a report from the Sociedad Espanola de Implantes World Congress consensus meeting in Barcelona, Spain. Clin. Implant Dent. Relat. Res., 5:57-60, 2003.

Armir, L. R.; Becking, A. G.; Jovanovic, A.; Perdijk, F., B., T.; Everts, V. \& Bronckers, A. L. J. J. Vertical distraction osteogenesis in the human mandible: a prospective morphometric study. Clin. Oral Implants Res., 17:41725, $2006 a$.

Armir, L. R.; Becking, A. G.; Jovanovic, A.; Perdijk, F. B., T.; Everts, V. \& Bronckers, A. L. J. J. Formation of new bone during vertical distraction osteogenesis of the human mandible is related to the presence of blood vessels. Clin. Oral Implants Res., 17:410-6, $2006 \mathrm{~b}$.

Bränemark, P. I. Osseointegration and its experimental background. J. Prosth. Dent., 50:399-410, 1983.

Brown, M. D. \& Hudlicka, O. Modulation of physiological angiogenesis in skeletal muscle by mechanical forces: involvement of VEGF and metalloproteinases. Angiogenesis, 2003;6(1):1-14, 2003.

Canalis, E.; McCarthy, T. \& Centrella, M. Growth factors and the regulation of bone remodeling. J. Clin. Invest., 81:27781, 1988.

Cecchinato, D. Olsson, C. \& Lindhe, J. Submerged or non submerged healing of endosseous implants to be used in the rehabilitation of partially dentate patients. J. Clin. Periodontol., 31:299-308, 2004.

Chiapasco, M.; Gratti, C.; Rossi, E.; Haefliger, W. \& Markwalder, T. H. Implant-retained mandibular overdentures with immediate loading. A retrospective multicenter study on 226 consecutives cases. Clin. Oral Impl. Res., 8:48-57, 1997. 
Chiapasco, M.; Gatti, C. \& Gatti, F. Immediate loading of dental implants placed in severely resorbed edentulous mandibles reconstructed with autogenous calvarial grafts. Clin. Oral Impl. Res., 18:13-20, 2007.

Cochran, D.; Morton, D. \& Weber, H. S. Consensus statements and recommended clinical procedures regarding loading protocols for endosseous dental implants. Int. J. Oral Maxillofac. Implants, 19(7):109$13,2004$.

Cortez, A. L. V.; Rabelo, G. O.; Mazzonetto, R. Reconstrução de maxila atrófica utilizando osso autógeno e malha de titânio para posterior reabilitação com implantes - caso clínico. Rev. Port. Estomatol. Med. Dent. e Cir. Maxilofac., 45(3):163-7, 2004.

Degidi, M.; Artese, L.; Rubini, C.; Perrotti, V.; Iezzi, G. \& Piattelli, A. Microvessel density and vascular endothelial growth factor expression in sinus augmentation using Bio-Oss®. Oral Disease, 12:469$75,2005$.

Degidi, M. \& Piattelli, A. A 7-year follow-up of 93 immediately loaded titanium dental implants. J. Oral Implants, 31(1):25-31, 2005.

Ericsson, I.; Nilson, H.; Lindh, T.; Nilner, K. \& Randow, $\mathrm{K}$. Immediate functional loading of Bränemark single tooth implants. An 18 month's clinical pilot follow-up study. Clin. Oral Impl. Res., 11:26-33, 2000.

Froum, S.; Cho, S. C.; Elian, N.; Rosenberg, E.; Rohrer, M. \& Tarnow, D. Extraction sockets and implantation of hydroxyapatites with membrane barriers. A histologic study. Implant. Dent., 13:153-64, 2004.

Gapski, R.; Wang, H. L.; Mascarenhas, P. \& Lang, N. P. Critical review of immediate implant loading. Clin. Oral Impl. Res. 14:515-27, 2003.

Grageda, E. Platelet-rich plasma and bone grafo materials: a review and standardized research protocol. Implant Dent., 13:301-9, 2004.

Gruber, R.; Baron, M.; Busenlechner, D.; Kandler, B.; Fuerst, G. \& Watzek, G. Proliferation and osteogenic differentiation of cells from cortical bone cylinders, bone particles from mill, and drilling dust. J. Oral Maxillofac. Surg., 63:238-43, 2005.

Hanson, S. \& Werke, M. The implant thread as a retention element in cortical bone: the effect of thread size and thread profile: a finite element study. J. Biomechanics, 36:1247-58, 2003.

Hing, K. A.; Wilson, L.F. \& Buckland, T. Comparative performance of three ceramic bone graft substitutes. The Spine Journal, 2007 (in press)

Hobkirk, J. A. \& Wiskott, H. W. A. Biomechanical aspects of oral implants. Consensus report of working group I. Clin. Oral Impl. Res., 17 (suppl. 2):52-4, 2006.

Horiuchi, N. \& Maeda, T. Statins and bone metabolism. Oral Diseases, 12:85-101, 2006.

Huang, Y. C.; Kaigler, D.; Rice, K.; Krebsbach, P. \& Mooney, D. Combined angiogenic and osteogenic factor delivery enhances bone marrow stromal cell-driven bone regeneration. J. Bone Miner. Res., 20: 848-57, 2005.

Issa, M. J. P.; Tiossi, R.; Pitol, D. L. \& Mello, S. A. TGF$\mathrm{b}$ and new bone formation. Int. J. Morphol., 24:339405, 2006a.

Issa, M. J. P.; Nascimento, C.; Barbosa, S. R. E.; Mello, S. A. \& Iyomasa, M. M. Morphogenetic protein rhBMP2 and new formation. Int. J. Morphol., 24:323-30, 2006b.

Jensen, S. S.; Broggini, N.; Hjorting-Hansen, E.; Schenk, R. \& Buser, D. Bone healing and graft resorption of autograft, anorganic bovine bone and b-tricalcium phosphate. A histologic and histomorphometric study in the mandibles of minipigs. Clin. Oral Implant. Res., 17: 237-43, 2005.

Kalmey, J. K. \& Lovejoy, C. O. Collagen fiber orientation in the femoral necks of apes and humans: do their histological structures reflect differences in locomotor loading?. Bone 31: 327-32, 2002.

Kawahara, H.; Kawahara, D.; Hayakawa, M.; Tamai, Y.; Kuremoto, T. \& Matsuda, S. Osseointegration Ander immediate loading: biomechanical stress-strain and bone formation-resorption. Implant Dent., 12: 61-8, 2003.

Kleinheinz, J.; Stratmann, U.; Joos, U. \& Wiesmann, H. P. VEGF-Activated angiogenesis during bone regeneration. J. Oral Maxillofac. Surg., 63:1310-6, 2005.

Kübler, A.; Neugebauer, J.; Oh, J. H.; Scheer, M. \& Zöller, J. Growth and proliferation of human osteoblasts on different bone graft substitutes. An in vitro study. Implant Dent., 13:171-9, 2004. 
Li, T. S.; Hamano, K.; Nishida, M.; Hayashi, M.; Ito, H.; Mikamo, A. \& Matsuzaki, M. (2003) CD117+ stem cells play a key role in therapeutic angiogênese induced by boné marrow cell implantation. Am. J. Physiol. Hearth. Circ. Physiol., 285:931-7, 2003.

Lienau, J.; Schell, H.; Duda, G.; Seebeck, P.; Muchow, S. \& Bail, H. Initial vascularization and tissue differentiation are influenced by fixation stability. J. Orthopaedic Res. 23:639-45, 2005.

Liu, Y.; Dulchavsky, D.; Gao, X.; Kwon, D.; Chopp, M.; Dulchavsky, S. \& Gautam, S. Wound repair by bone marrow stromal cells through growth factor production. J. Surg. Res., 136:336-41, 2006.

Lorenzoni, M.; Pertl, Ch.; Zhang, K.; Wimmer, G. \& Wegscheider, W. A. Immediate loading of singletooth implants in the anterior maxilla. Preliminary results after one year. Clin. Oral Impl. Res., 14:18087, 2003.

Madeddu, P. Therapeutic angiogenesis and vasculogenesis for tissue regeneration. Exp. Physiol., 90(3):315-26, 2005.

McMillan, P. J.; Riggs, M. L.; Bogle, G. \& Crigger, M. Variables that influence the relationship between osseointegration and bone adjacent to an implant. Int. J. Oral Maxillofac Implants, 15:654-61, 2000.

Meyer, U.; Wiesmann, H. P. Fillies, T. \& Joos, U. Early tissue reaction at the interface of immediately loaded dental implants. Int. J. Oral Maxillofac. Implants, 18: 489-99, 2003.

Miura, M.; Miura, Y.; Sonoyama, W.; Yamaza, T.; Gronthos, S. \& Shi, S. Boné marrow-derived mesenchymal stem cells for regenerative medicine in craniofacial region. Oral Disease, 12:514-22, 2006.

Moghadam, H.; Sándor, G.; Colmes, H. \& Clokie, C. Histomorphometric evaluation of bone regeneration using allogeneic and alloplastic boné substitutes. J. Oral Maxillofac. Surg., 62:202-13, 2004.

Moore, W. R.; Graves, S. E. \& Bain, G. I. Synthetic bone substitutes. A. N. Z. J. Surg., 71:354-61, 2001.

Morton, D.; Jaffin, R. \& Weber, H. P. Immediate restoration and loading of dental implants: clinical considerations and protocols. Int. J. Oral Maxillofac. Implants, 19 (suppl):103 -8, 2004.
Nishimura, I.; Shimizu, Y. \& Ooya, K. Effects of cortical bone perforation on experimental guided bone regeneration. Clin. Oral Implant. Res., 15:293-300, 2004.

Nkenke, E.; Lehner, B.; Weinzierl, K.; Thams, U.; Neugebauer, J.; Steveling, H.; Radespiel-Tröger, M. \& Neukam, F., W. Bone contact, growth, and density around immediately loaded implants in the mandible of mini pigs. Clin. Oral Impl. Res., 14:312-21, 2003.

Nkenke, E.; Lehner, B.; Fenner, M.; San Roman, F.; Thams, U.; Neukam, F. W. \& Radespiel-Tröger, M. Immediate versus delayed loading of dental implants in the maxillae of minipigs: follow-up of implant stability and implant failures. Int. J Oral Maxillofac. Implants, 20:39-47, $2005 \mathrm{a}$.

Nkenke, E.; Fenner, M.; Vairaktaris, E., G.; Neukam, F., W. \& Radespiel-Tröger, M. Immediate versus delayed loading of dental implants in the maxillae of minipigs. Part II: histomorphometric analysis. Int. J. Oral Maxillofac. Implants 20:540-6. 2005b.

Nkenke, E. \& Fenner, M. Indications for immediate loading of implants and implant success. Clin. Oral Impl. Res., 17 (suppl. 2):19-34, 2006.

Olate, S.; Alister, J. P.; Soto, M.; Alveal, R.; Fuentes, J. \& Thomas, D. Extracciones e indicaciones de extracciones dentales en población rural chilena de 11 a 30 años. Av. Odontoestomatol., 22 (2):119-24, 2006.

Olmedo, D.; Fernández, M. M.; Guglielmotti, M. B. \& Cabrini, R., L. (2003) Macrophages related to dental implant failure. Implant Dent., 12:75-80, 2003.

Ottoni, J. M. P.; Oliveira, Z. F. L.; Manzini, R. \& Cabral, A. M. Correlation between placement torque and survival of single-tooth implants. Int. J. Oral Maxillofac. Implants, 20:769-76, 2005.

Petersen, P. E. \& Yamamoto, T. Improving the oral health of older people: the approach of the WHO Global Oral Health Programme. Community Dent. Oral Epidemiol., 33:81-92, 2005.

Piattelli, A.; Degidi, M.; Di Stefano, D.; Rubini, C.; Fioroni, M. \& Strocchi, R. Microvessel density in alveolar ridge regeneration with autologous and alloplastic bone. Implant Dent., 11 (4):370-5, 2002.

Queiroz, T. P.; Hochuli-Vieira, E.; Gabrielli, M. A., C. \& Cancian, D. C. J. Use of bovine graft and boné membrane 
in defects surgically created in the cranial vault of rabbits. Histologic comparative analysis. Int. J. 2006.

Roethy, W.; Fiehn, E.; Suehiro, K.; Gu, A.; Hua, Yi, G.; Shimizu, J.; Wang, J.; Zhang, G.; Ranieri, J.; Akella, R.; Funk, S.; Sage, H.; Benedict, J. \& Burkhoff, D. A growth factor mixture that significantly enhances angiogenesis in vivo. J. P. E. T., 299:494-500, 2001.

Rosenkilde, M. \& Schwartz, T. The chemokine system - a major regulator of angiogenesis in health and disease. APMIS, 112:481-95, 2004.

Scarano, A.; Degidi, M.; Iezzi, G.; Petrone, G. \& Piattelli, A.Correlation between implant stability quotient and bone-implant contact: a retrospective histological and histomorphometrical study of seven titanium implants retrieved from humans. Clin. Implant Dent. Relat. Res. 8(4):218-22, 2006.

Schincaglia, G. P.; Marzola, R.; Scapoli, C. \& Scotti, R. Immediate loading of dental implants supporting fixed partial dentures in the posterior mandible: a randomized controlled split - mouth study - machined versus titanium oxide implant surface. Int. J. Oral Maxillofac. Implants, 22:35-46, 2007.

Schroeder, A.; Maeglin, B. \& Sutter, F. Das ITIHohlzylinderimplantat Typ F zur Prothesenretention beim zahnlosen Kiefer. Schweizerische Monatschrift fur Zahnheilkunde. 93:720-33, 1983.

Schwarz, F.; Rothamel, D.; Herten, M.; Sager, M. \& Becker, J. Angiogenesis pattern of native and cross-linked collagen membranes: an immunohistochemical study in the rat. Clin. Oral Implant Res., 17:403-9, 2006.

Serra e Silva, F. M.; Albergaria-Barbosa, J. R. \& Mazzonetto, R. Clinical evaluation of association of bovine organic osseous matrix and bovine bone morphogenetic protein versus autogenous bone graft in sinus floor augmentation. J. Oral Maxillofac. Surg., 64:931-5, 2006.

Suba, Z.; Takács, D.; Matusovits, D.; Barabás, J.; Fazekas, A. \& Szabó G. Maxillary sinus floor grafting with btricalcium phosphate in humans: density and microarchitecture of the newly formed bone. Clin. Oral Impl. Res., 17:102-8, 2006.

Szmukler-Moncler, S.; Piatelli, A.; Favero, G., A. \& Dubruille, J. H. Considerations preliminary to the application of early and immediate loading protocols in dental implantology. Clin. Oral Impl. Res., 11:12-25, 2000.
Tamimi, F. M.; Torres, J.; Tresguerres, I.; Clemente, C.; López-Cabarcos, E. \& Blanco, L. J. Bone augmentation in rabbit calvariae: comparative study between Bio-Oss ${ }^{\circledR}$ and a novel b-TCP/DCPD granulate. J. Clin. Periodontol., 33:922-8, 2006.

Thorwarth, M.; Schultze-Mosgau, S.; Kessler, P.; Wiltfang, J. \& Schlegel, K. A. Bone regeneration in osseous defects using a resorbable nanoparticular hydroxyapatite. J. Oral Maxillofac. Surg., 63:1626-33, 2005.

Traini, T.; Degidi, M.; Caputi, S.; Strocchi, R.; Di lorio, D. $\&$ Piattelli, A. Collagen fiber orientation in human periimplant boné around immediately loaded and unloaded titanium dental implants. J. Periodontol., 76:83-9, 2005.

Urist, M., R.; Delange, J., R. \& Finerman, G. A. M. Bone cell diferentiation and growth factors. Science, 220: 6806, 1983.

Wang, H. L.; Ormianer, Z.; Palti, A.; Perel, M.; Trisi, P. \& Sammartino, G. Consensus conference on immediate loading: the single tooth and partial edentulous areas. Implant. Dent., 15:324-33, 2006.

Weiss, S.; Zimmermann, G.; Baumgart, R.; Kasten, P.; Bidlingmaier, M. \& Henle, P. Systemic regulation of angiogenesis and matrix degradation in bone regeneration--distraction osteogenesis compared to rigid fracture healing. Bone, 37(6):781-90, 2005.

Winet, H. The role of microvasculature in normal and perturbed bone healing as revealed by intravital microscopy. Bone 19 (supp 1): 39S-57S, 1996.

Dirección para correspondencia:

Prof. Dr. Jose Ricardo de Albergaria Barbosa

Av. Limeira 901

Caixa Postal 52

CEP 13414-903

Piracicaba - SP

BRASIL

Tel.: (19) 2106-5200

Fax.: (19) 3421-0144

Email: barbosa@fop.unicamp.br

Recibido : 11-05-2007

Aceptado: 15-06-2007 
